\section{BTh 04}

HEAD AND NECK CANCER - TREATMENT BY PERMANENT AND/OR REMOVABLE INTERSTITIAL RADIOACTIVE IMPLANTS

Don R. Goffinet, M.D.

High dose interstitial implant boosts may be delivered by either removable (192-Iridium) or permanent (125-Iodine, 198-Gold) sources. Such procedures not only deliver a high radiation dose to the tumor volume but also reduce the risk of normal tissue complications. More than 1000 interstitial implants have been performed at Stanfard since 1975. The various techniques used for implanting both primary sites (tonsil-palate and base tongue), and the carotid region intra-operatively for massive cervical lymphadenopathy will be discussed.

65 patients with oropharyngeal malignancies have been treated either by radiation therapy and implantation or by surgery and post-operative irradiation. An $86 \%$ local control rate has been obtained in 22 patients with base tongue cancers when external beam irradiation and removable 192-Ir seed implantation have been combined. Almost $80 \%$ of these patients remain without evidence of disease (NED). Only $27 \%$ of the patients managed by surgery and radiation therapy, without implantation, remain NED and these patients have experienced significantly more cervical lymph node relapses than the group managed by external beam irradiation and implantation. In another 28 patients with tonsillo-palatine carcinomas who were treated by $192-$ Ir implants, the primary site was controlled in $26(93 \%)$ Removable interstitial implant boosts have resulted in a high local control rate in these patients with few complications.

43 other patients underwent radical neck dissection and simultaneous 125-Iodine seed carotid artery region inplantation via absorbable suture (vicry1) carriers. A local control rate of $80 \%$ was obtained in these patients, all of whot had massive cervical 1 ymph node involvement, and little whom had massive cervical lymph node involvement, and little The techniques of implantation, radiation dose distributions and volumetric aspects of dosimetry will also be presented.

Radiation Therapy Division, Stanford University School of Medicine, Stanford, California 94305

\section{BTh 05}

CLINICAL EXPERIENCE WITH IODINE-125-SEEDS IN LOCALLY ADVANCED TUMORS OF THE GI-TRACT

J.R. Siewert and 0.H. Bertermann

The majority of patients with far advanced gastrointestinal tumors are not curative resectable. The need for a meaningful palliation remains a challenge. Morbidity with palliative treatment - high-dose external radiotherapy, chemotherapy or by-pass procedures- is high. The objective of this study is to report on our own preliminary data. Between 1984 and 1985 in 20 patients Iodine125 -seeds were implanted. All patients had locally advanced GI-tract tumors. Median age was 58 years. Our series included carcinoma of : pancreas $(n=5)$, esophagus $(n=3)$, sto$\operatorname{mach}(n=8)$, rectum $(N=2), \operatorname{col} n(n=1)$ and bileduct $(n=1)$. Implantation was performed by a Mick-applicator or seeds in carrier. The morbidity with implantation in pancreatic carcinoma was high. Rupture of a transverso transversostomy and a pancreatic fistula as well as a pancreatic abscess fid occur within the area of the implantation procedure. In all of the patients a marked improvement in pain control could be documented. Furthermore, a. good local control of tumor growth was achieved for a short period. The question remains if implantation of Iodine-125 improves survival time cannot be answered presently due to a limited observation time. However five patients did have a survival of greater than eight month.

In summary implantation of pancreatic carcinoma is accompanied by a high morbidity. However in the majority of patients a good palliative effect could be achieved. For those patients with only a short life expectancy the implantation with Iodine-125-seeds may markedly improve their quality of life.

Chirurgische Klinik und Poliklinik der Technischen Universität München, KIinikum rechts der Isar, IsmaningerStr. 22, 8000 München 80
BTh 06

TREATMENT OF SOFT TISSUE SARCOMA OF LIMBS BY FUNCTIONSAVING RESECTION AND BRACHYTHERAPY.

M. H. Shiu and B. S. Hilaris.

Soft tissue sarcomas (STS) tend to recur local1y after inadequate excision. Wide resect ion is needed for complete surgical extirpation, but this often leads to major sacrifice of function. Function-saving resection (FSR) and brachytherapy (BRT) of the tumor-bed have been applied to the management of locally advanced STS of the limbs in 33 patients (pts.). Surgical excision was performed so that adjacent major artery, vein, nerve, bone or joint would be preserved. A wide margin of resection was thus not obtained, and microscopic or minimal $\mathrm{v}$ is ible tumor was left behind if necessary to preserve function. Nylon catheters were inserted percutaneously into the tumor-bed during surgery, for loading of iridium-192 sources usually 72 hours or latex. The standard dose was 1,000 cGy per day for 4-5 days as measured at one corner of the implant $0.5 \mathrm{~cm}$ from its plane. Supp lementary external radiation therapy (ERT) was given in 7 pts. $18 \mathrm{pts}$. received ad juvant chemotherapy using adriamyc in and other drugs. Results with 4 to 10 year follow-up showed successful local control in 16 of 17 previous $1 y$ untreated and 10 of 16 previous $1 y$ treated, locally recurrent sarcomas. 3 of the 7 local failures were saIvaged by further excision and BRT/ERT. Metastases developed in 7 pts.; 6 died. Comp 1 ications include delayed wound healing requiring skin graft, 4 pts., wound necrosis requiring amputation, 2 pts., and chronic sinus, 2 pts. Good to excel lent function was achieved in $26(79 \%)$ of the 33 pts.: all could walk on the treated lower $1 \mathrm{imb}$ or use the treated upper limb for prehensile purposes. These results indicate that FSR and BRT +/- ERT can offer good local control of STS in a

1 imb. Comp 1 ications in recent years have been reduced by later loading and the use of thick, viable wound flaps.

Memorial Sloan-Rettering Cancer Center, 1275 York Avenue, New York, N.Y. 10021, U.S.A.

\section{BTh 07}

THE PALLIATVE HIGH DOSE RATE A FTERLOA DINGTHERA PY IN COMBINATION WITH ENDOSCOPIC IASER TREA TMENT OF MALIEN SPENOSES OF THE UPPER GA STROINTESTIKAL TRACT M.Bader, R.J.Dittler, G.Ries and J.R. Siewert

60 patients suffering from malign stenoses of the esophagus: and the esophago-cardial junction were submitted to endocavitary afterloading-therapy after laserendoscopic recanalisation.

An intraluminary tumor reduction and fast elimination of dysphagia was possible.This method provides an alternative to endoscopic tubus-1mplantation.

The afterloading-probe is placed endoskopically under vision. With a 192-Iridium radiation source 7 Gy were applied in $1 \mathrm{~cm}$ distance from the probe. A permanent relief from dysphagic symptoms was achieved in $77 \%$, in $23 \%$ a restenosis during therapy was observed.

The mean survival time was 6 months.

The method shows a low incidence of complications, it can be used in out-patients and in combination with percutaneous radiation therapy as boostradiation.

Chirurgische Klinik und Poliklinik und Institut für Radiologische Onkologie und Strahlentherapie der Technischen Universit at München, Ismaningerstr.22 D 8000 Minchen 80 F.R.Germany 